\title{
Molecular gas and dust emission in a $z=3.63$ strongly lensed starburst merger at sub-kiloparsec scales
}

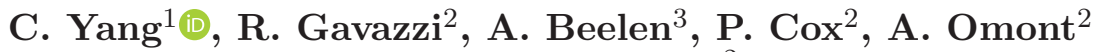 \\ and M. D. Lehnert ${ }^{2}$ \\ ${ }^{1}$ European Southern Observatory, Alonso de Córdova 3107, \\ Vitacura, Santiago, Chile \\ email: cyang@eso.org \\ ${ }^{2}$ Institut d'Astrophysique de Paris, Sorbonne Université, CNRS (UMR 7095), \\ 98 bis bd Arago, 75014 Paris, France \\ ${ }^{3}$ Institut d'Astrophysique Spatiale, CNRS (UMR 8617), \\ Université Paris-Sud, Université Paris-Saclay, Orsay, France
}

\begin{abstract}
We present 0.'2-0.'4 resolution ALMA images of the submillimeter dust continuum and the $\mathrm{CO}, \mathrm{H}_{2} \mathrm{O}$, and $\mathrm{H}_{2} \mathrm{O}^{+}$line emission in a $z=3.63$ strongly lensed dusty starburst. We construct the lens model for the system with an MCMC technique. While the average magnification for the dust continuum is about 11, the magnification of the line emission varies from 5 to 22 across the source, resolving the source down to sub-kpc scales. The ISM content reveals that it is a pre-coalescence major merger of two ultra-luminous infrared galaxies, both with a large amount of molecular gas reservoir. The approaching galaxy in the south shows no apparent kinematic structure with a half-light radius of $0.4 \mathrm{kpc}$, while the preceding one resembles a $1.2 \mathrm{kpc}$ rotating disk, separated by a projected distance of $1.3 \mathrm{kpc}$. The distribution of dust and gas emission suggests a large amount of cold ISM concentrated in the interacting region.
\end{abstract}

Keywords. galaxies, high-redshift, ISM, gravitational lensing, submillimeter, molecules

\section{Introduction}

The strongest starbursts across cosmic time are the heavily dust-obscured submillimeter galaxies (SMGs, see e.g. Casey et al. 2014). With total infrared (IR) luminosities above $10^{12} L_{\odot}$, SMGs reach the limit of "maximum starbursts" (Barger et al. 2014) with star formation rates exceeding $1000 M_{\odot} \mathrm{yr}^{-1}$. Such intense star formation is thought to be triggered by galaxy mergers or at least enhanced by interactions, which is consistent with $\Lambda$ CDM simulations where merger rates are expected to increase with increasing redshift (e.g. Rodriguez-Gomez et al. 2015), although there are also pieces of evidence that some of the SMGs are rather smooth isolated clumpy disks with high gas fractions (e.g. Hodge et al. 2019). The nature of the SMGs remains hotly debated, in part because of the details of the structure of the molecular gas and dust content (fuel of star formation). Therefore, to better understanding the nature of SMG, we conduct a detailed study of $\mathrm{CO}$ and $\mathrm{H}_{2} \mathrm{O}$ (which is a powerful diagnostic tool of the far-IR radiation fields, Yang et al. 2016) and dust content in a strongly lensed SMG selected from the Herschel-ATLAS survey (Bussmann et al. 2013), G09v1.97 (Yang et al. 2019), down to the sub-kpc scales, with the help of gain sensitivity and angular resolution from lensing magnification. 
G09v1.97, $z=3.63$

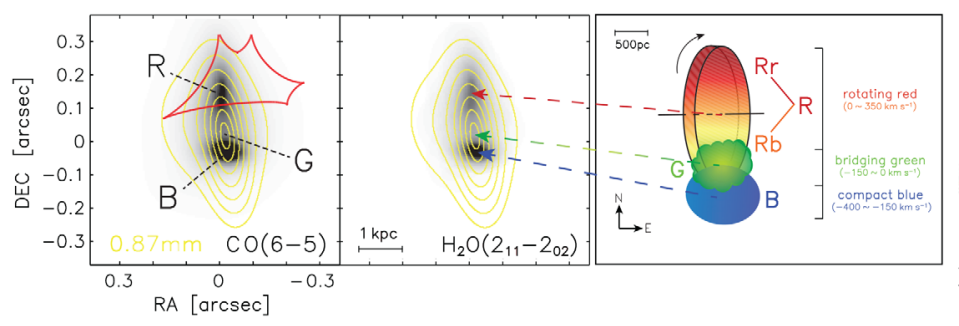

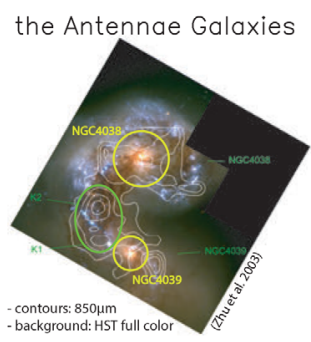

Figure 1. The reconstructed image in the source plane, from left to right: the moment-0 map of $\mathrm{CO}(6-5)$, with yellow contours of the $188 \mu \mathrm{m}$ dust emission in the rest-frame; the moment-0 map of $\mathrm{H}_{2} \mathrm{O}\left(2_{11}-2_{02}\right)$, with similar contours; the sketch of the merging scenario of G09v1.97; the optical image with white contours showing the $850 \mu \mathrm{m}$ SCUBA image of the Antenna Galaxies (Zhu et al. 2003).

\section{Results}

We have conducted ALMA observations of the $\mathrm{CO}(6-5), \mathrm{H}_{2} \mathrm{O}\left(2_{11}-2_{02}\right), \mathrm{H}_{2} \mathrm{O}^{+}$ $\left(2_{02}-1_{11}\right), \mathrm{H}_{2} \mathrm{O}^{+}\left(2_{11}-2_{02}\right)$ and the dust continuum emission, resulted in high signal-tonoise data-cubes with synthesis beam-sizes around $0.2-0$.'4. The dust continuum and molecular gas emission are resolved into a nearly complete $\sim 1^{\prime \prime} .5$ diameter Einstein ring plus a weaker image in the centre, which is caused by a special dual deflector lensing configuration. The velocity structures of the three lines in the image plane are strikingly similar.

Line profile. The (image plane) spatially integrated, continuum-subtracted spectra of $\mathrm{CO}(6-5), \mathrm{H}_{2} \mathrm{O}\left(2_{11}-2_{02}\right)$, and the $J_{\text {up }}=2 \mathrm{H}_{2} \mathrm{O}^{+}$have the same profile, which is composite of three Gaussian components, a distinct blue (B) and two neighbouring red ones ( $\mathrm{Rr}$ and $\mathrm{Rb}$ ). This similarity suggests these three tracers are co-spatially located, tracing the warm dense gas that directly relates to the intense star formation.

Lens modelling. In order to derive the intrinsic properties of G09v1.97, a lens model needs to be built. Using the 0 '2 ALMA image of the dust continuum of the background source and KECK images of the two foreground deflectors, we build a double-SIE lens model with an MCMC approach. Then the lens model is applied to the line emissions per channel to reconstruct the emission in the source plane.

The nature of the SMG in the source plane. The reconstructed source-plane image of the $\mathrm{CO}(6-5)$ and $\mathrm{H}_{2} \mathrm{O}\left(2_{11}-2_{02}\right)$ line are similarly showing a bimodality - the northern component is mainly associated with the two red-shifted Gaussian components seen in the spectrum, while the southern component is dominantly from the blue-shifted spectral components. As shown in Fig 1, the approaching galaxy B shows no apparent kinematic structure with a semi-major half-light radius of $a_{\mathrm{S}}=0.4 \mathrm{kpc}$, while the receding galaxy $\mathrm{R}$ resembles an $a_{\mathrm{s}}=1.2 \mathrm{kpc}$ rotating disk. The dust emission is best modelled with two components, a compact cold dust $(17 \mathrm{~K})$ dust component peaking in between $\mathrm{R}$ and $\mathrm{B}$, and an extended warmer dust $(33-61 \mathrm{~K})$ component dominated by the $\mathrm{R}$ and $\mathrm{B}$ galaxies. The two galaxies are separated by a projected distance of $1.3 \mathrm{kpc}$, bridged by weak line emission that is co-spatially located with the cold dust emission peak in the centre, suggesting a large amount of cold interstellar medium (ISM) in the interacting region, much like the concentration of cold dust emission found in the Antenna Galaxies.

\section{References}

Barger, A. J., Cowie, L. L., Chen, C.-C., et al. 2014, ApJ, 784, 9

Bussmann, R. S., Pérez-Fournon, I., Amber, S., et al. 2013, ApJ, 779, 25 
Casey, C. M., Narayanan, D., \& Cooray, A. 2014, Phys. Rep., 541, 45

Hodge, J. A., Smail, I., Walter, F., et al. 2019, ApJ, 876, 130

Rodriguez-Gomez, V., Genel, S., Vogelsberger, M., et al. 2015, MNRAS, 449, 49

Tacconi, L. J., Genzel, R., Neri, R., et al. 2010, Nature, 463, 781

Yang, C., Omont, A., Beelen, A., et al. 2016, A\&A, 595, A80

Yang, C., Gavazzi, R., Beelen, A., et al. 2019, A\&广A, 624, A138

Zhu, M., Seaquist, E. R., \& Kuno, N. 2003, ApJ, 588, 243 